\title{
The Zemblanity of Missing Subtile Changes in the Patient'S Blood Tests-Diagnosing Hereditary Spherocytosis After Finding Extramedullary Haematopoiesis
}

\author{
Margarida Dantas Brito*1, Harald Holte ${ }^{1}$ and Irene Langmyhr ${ }^{2}$ \\ ${ }^{1}$ Lymphoma department, Oslo University Hospital, Norway \\ ${ }^{2}$ Patology department, Oslo University Hospital, Norway
}

Received: March 08, 2018; Published: March 19, 2018

*Corresponding author: Ana Margarida Dantas Brito Rodrigues, Lymphoma department, The Norwegian Radium Hospital, Oslo University Hospital, Norway.

\section{Abstract}

We report a clinical case of hereditary spherocytosis in the view of it's educational value. The diagnose was overlooked for many years while the patient was a regular blood donor. This was probably the stress factor that precipitated the development of extramedullary haematopoiesis in a patient that otherwise had a rather mild phenotype. The authors want to stress the importance of valuing the red cell indexes as an informative part of the hemogram.

Keywords: Hereditary Spherocytosis; Red Blood Cell Indexes; Mean Corpuscular Haemoglobin Concentration (MCHC); Haemolysis; Extramedullary Haematopoiesis

\section{Introduction}

A 59-year old female patient was referred to the lymphoma department. The patient's previous medical history was unremarkable. Symptoms started after she had an accidental fall on her back, and she was in need of analgesics for some time because of back pain. Eventually she consulted her family physician who ordered a thorax CT scan suspecting a spine fracture. The CT scan showed several para vertebral enlarged lymph nodes, the biggest measured $3,4 \times 1,6 \times 3,2 \mathrm{~cm}$. Additionally she had radiological splenomegaly $(15 \times 15 \times 15 \mathrm{~cm})$. She was then referred to the lymphoma department. The referral document reported normal blood tests. At the first appointment she was in god shape, the pain had gradually improved, and she had no B symptoms, palpable peripheral lymph nodes nor splenomegaly. The blood tests were described as overall normal apart from elevated total bilirubin (52凶mol/L, reference range 5-25) and elevated lactate dehydrogenase (267 U/L, reference range 105-205). She was referred to an ultrasound-guided lymph node core biopsy and bone marrow biopsy.

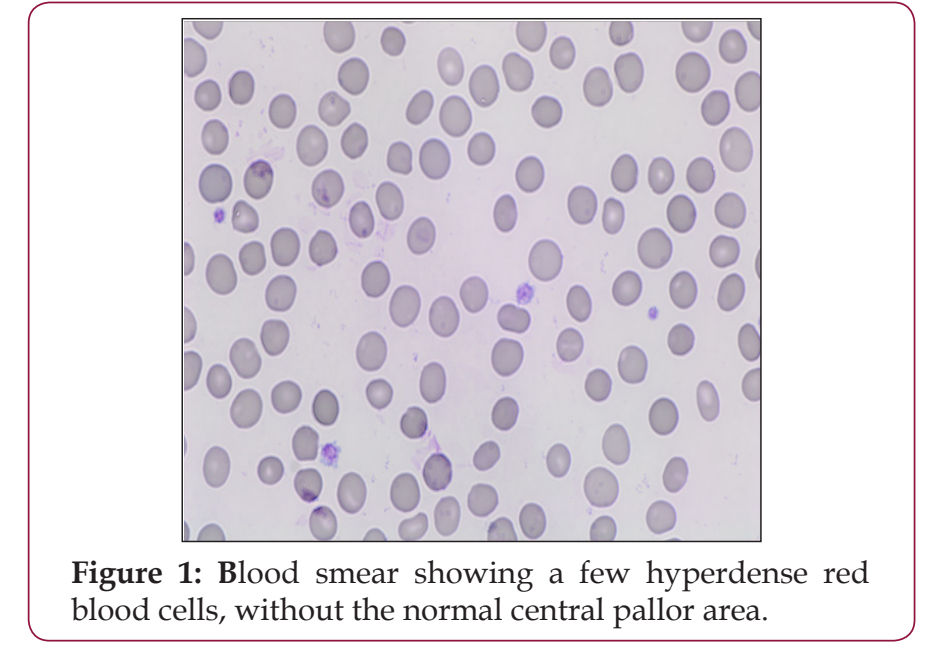

The histology from the lymph node revealed three-linear extramedullary haematopoiesis, with no other signs pathology. The bone marrow biopsy showed mildly elevated cellularity, with 
no signs of pathology. Importantly there were no signs of fibrosis or myeloproliferative diseases, that at this point were considered a differential diagnose in the view of the patient's age and the surprising finding of extramedullary haematopoiesis. After these results we review the blood tests and it was evident that the patient had chronic compensated haemolysis. In addition to the findings initially reported in the medical journal, the blood tests taken at admission showed reticulocytosis (330 x 109/L, reference range 30-100) and very low haptoglobin $(<0.1$, reference range $0,4-2,1$ $\mathrm{g} / \mathrm{L}$ ). The haemoglobin was $14 \mathrm{~g} / \mathrm{dL}$, MCHC was $35 \mathrm{~g} / \mathrm{dL}$ (reference range 31-35) and the RDW was 18\% (reference range 11-15). In a second appointment we reviewed the clinical history and ordered new blood tests. The patient hadn't had known anaemia previously. In fact, she said she had been a regular blood donor for more than 20 years (Figure 1).

She stopped donating blood only a couple of years ago, because of lack of time. On further examination, she revealed to have an adult son with a mild anaemia because of hereditary spherocytosis. He was diagnosed in early childhood during what seems like an aplastic crisis episode. There are no other known family members with this diagnosis. The second set of blood tests revealed haemoglobin 12,7 g/L, MCHC $37 \mathrm{~g} / \mathrm{dL}$, reticulocytosis $250 \times 109$ $/ \mathrm{L}$, haptoglobin $<0,1 / \mathrm{L}$, total bilirubin $53 \otimes \mathrm{mol} / \mathrm{L}$, with normal ASAT and ALAT, lactate dehydrogenase $222 \mathrm{U} / \mathrm{L}$ and the direct antiglobulin test (Coombs test) was negative. The blood smear revealed spherocytosis. The eosin-5'-maleimide (EMA) binding test was compatible with hereditary spherocytosis (HS). The abdominal echography showed no signs of gallstones. We prescribed folate supplementation (1mg/day) and informed the patient that she is not suitable as blood or bone marrow donor and contacted the bone marrow donors register (Figure 2).

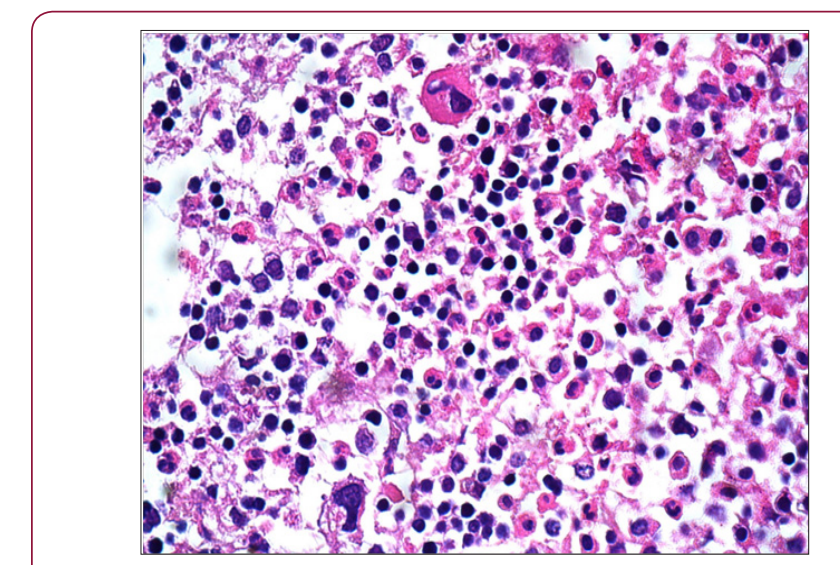

Figure 2: H\&E stained section shows megakaryocytes, erythroblasts, and immature myeloid elements.

The patient's daughter will be screened for HS. We next sought for old blood tests to see if there have been signs of haemolysis previously overlooked. The patient said she has taken blood tests mostly in relation with blood donation. We had access to the blood tests taken in the blood bank (several analyses taken between 1996 and 2014), but unfortunately these were much directed tests only with four parameters: haemoglobin, platelets, ferritin and ALAT. The family doctor had ordered blood tests taken in 2012 showed haemoglobin 13,5 g/dL, normal MCV and MCH, no report of MCHC, folate deficiency $(3,6 \mathrm{nmol} / \mathrm{L}$, reference range $>5,7)$, elevated total bilirubin ( $31 \mathrm{umol} / \mathrm{L}$, reference range $<26$ ), elevated lactate dehydrogenase 218 (reference range $<205 \mathrm{U} / \mathrm{L}$ ).

\section{Discussion}

This clinical case has an unusual clinical presentation and a delayed diagnose of HS. The authors report it in the view of its educational value. Mild cases of HS are often well compensated, with no signs of anaemia. Diagnosing this subclinical forms can be very challenging [1,2]. Mild clinical cases often don't warrant any treatment beside folate supplementation [3] for some patients. Nevertheless, it can be important to diagnose these patients since they may be prone to late complications as gallstone disease or splenomegaly. Furthermore, it is important to know the diagnosis because haemolysis can be exacerbated by acute illnesses, infectious mononucleosis, superimposed megaloblastic anaemia due to an unmet increased need for folate, or by other factors as pregnancy or exercise $[1,3]$ It is therefore recommended that after diagnosis of a patient with HS, family members should be examined [1] The literature indicates autosomal dominant inheritance in approximately $75 \%$ of patients [1-3].

Despite having a son with HS and having findings that could have raised the suspicion on haemolysis in her previous "routine" blood tests, the patient was never tested for this condition and she was not aware of the possibility she might be affected too. The red cells of these patients have a compromised membrane? Resistance and elastic deformability because of alterations in genes that encode proteins involved in the red cell's membrane. They have chronic haemolysis due to red cell membrane instability and conditioning by the spleen, leading to a stress compensatory haematopoiesis [3]. Naturally HS patients are not eligible as blood donors. Our patient had served as a blood donor for more than 20 years. Luckily enough she has never had known anaemia despite giving blood regularly. Extramedullary haematopoiesis is reported as a rare complication of HS $[4,5]$. One can speculate whether donating blood was the additional stress factor that precipitated the extramedullary haematopoiesis. On the other hand, HS patients have osmotically fragile red cells and are therefore unsuitable for transfusion from the recipient point of view. We found a publication about safety of blood bank storage of HS red blood cells.

This study was done with the aim of evaluating the feasibility of elective autologous transfusion in HS patients, after planned surgery. The authors concluded that HS red blood cells loose membrane even under optimum storage conditions and are unsuitable for autologous transfusion [6]. We want to stress the importance of valuing the red blood cell indexes reported by common automatic cell counters. A high RDW and MCHC should raise suspicion about $\mathrm{HS}$, particularly when there is also elevation of the bilirubin and lactate dehydrogenase. The combination of elevated MCHC and RDW is described to have a sensitivity of 63 $\%$ and a specificity of $100 \%$ for the diagnosis of HS [7,8]. Our laboratory serves a specialized oncology hospital and chooses not to report RDW in the standard hemogram. The result can be made 
available if the physician asks for it. In most general hospitals both RDW and MCHC are commonly reported as a part the hemogram. It is particularly relevant to consider this red cell indexes as a good screening tool in areas of high hereditary spherocytosis prevalence, as Scandinavia is. Indeed this is the most common disorder of the red cell membrane and the most common cause of inherited chronic haemolysis in northern Europe, where it affects about one person in 2000 [1].

Other important hints to mild HS diagnosis are often available in basic blood chemistry, as elevated bilirubin (indirect) and elevated lactate dehydrogenase. Aspartate aminotransferase can be slightly elevated. In the absence of liver disease this warrants investigation of haemolytic diseases. Pseudohyperkalemia can be also present as a subtle sign of HS, because of a high rate of potassium leakage out of the red cells [9]. The blood smear reveals spherocytes in most of these patients $[10,11]$. These are small, dark, dense hyperchromic red cells, without central pallor. While suggestive of hereditary spherocytosis, spherocytes can be seen in other contexts as autoimmune haemolytic anaemia $[10,11]$. The literature describes that spherocytes are not visible in about $2 \%$ of patients with mild HS [12]. All doctors aspire to perform an accurate diagnosis. It is often easy make the correct diagnose in retrospect. Unfortunately, diagnoses that need a high suspicion index are easily overseen in a busy clinical practise. The hemogram is probably the most frequently ordered medical test, and it can be the first lead to an unsuspected diagnosis. This is the case of many patients with chronic lymphatic leukaemia and chronic myeloid leukaemia that are nowadays commonly diagnosed by serendipity while still asymptomatic. On the contrary, failing to interpretate subtle yet persistent abnormalities in the hemogram or basic blood chemistry can later on give doctors a zemblanity moment, with the unpleasant discovery of something previously neglected.

\section{Conclusion}

This case is draws our attention to the importance of value the red cell indexes. One should pursue further investigation when persistent and unexplained abnormalities are found over time. It is often helpful to take contact with the laboratory staff and the haematologists to discuss the hemogram findings.

\section{References}

1. Perrotta S, Gallagher PG, Mohandas N (2008) Hereditary spherocytosis, Lancet 372(9647): 1411-1426.

2. Bolton Maggs PH, Langer JC, Iolascon A, Tittensor P, King MJ (2011) Guidelines for the diagnosis and management of hereditary spherocytosis-2011 update, Br J Haematol 156(1): 37-49.

3. Rencic J, Zhou M, Hsu G, Dhaliwal G (2017) Circling Back for the diagnosis. N Engl J Med 377(18): 1778-1784.

4. Kim CH (2010) Homeostatic and pathogenic extramedullary hematopoiesis. J Blood Med 1: 13-19.

5. Smith J, Rahilly M, Davidson K (2011) Extramedullary haematopoiesis secondary to hereditary spherocytosis. Br J Haematol 154(5): 543.

6. Weinstein R, Martinez R, Hassoun H, Palek K (1997) Does a patient with hereditary sphefocytosis qualify for preoperative autologous blood donation? Transfusion 37(11-12): 1179-1183.

7. Michaels LA, Cohen AR, Zhao H, Raphael RI, Manno CS (1997) Screening for hereditary spherocytosis by use of automated erythrocyte indexes. J Pediatr 130(6): 957-960.

8. Failace R Hemogram (2009) $5^{\text {th }}$ (Edn.). (portuguese version), Artmed (Eds.). São Paulo, Brazil.

9. Alani FS, Dyer T, Hindle E, Newsome DA, Ormerod LP, et al. (1994) Pseudohyperkalaemia associated with hereditary spherocytosis in four members of a family. Postgrad Med J 70(828): 749.

10. Bain B (2005) Diagnosis from the blood smear. Engl J Med 353(5): 498507.

11. Palek K, Jarolim P (1993) Clinical expression and laboratory detection of red blood cell membrane protein mutations. Semin Hematol 30(4): 249.

12. Mariani M, Barcellini W, Vercellati C, Marcello AP, Fermo E, et al. (2008) Clinical and hematologic features of 300 patients affected by hereditary spherocytosis grouped according to the type of the membrane protein defect. Haematologica 93(9): 1310-1317.
This work is licensed under Creative Commons Attribution 4.0 License

Submission Link: https://biomedres.us/submit-manuscript.php

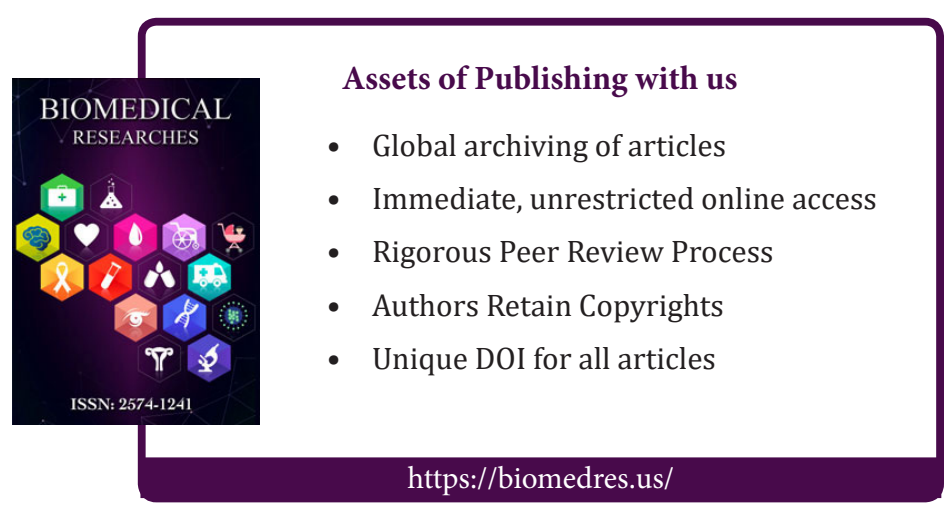

